\title{
A case of Galloway-Mowat syndrome with novel compound heterozygous variants in the WDR4 gene
}

\author{
Hamin Kim ${ }^{\oplus}$, Hyunjoo Lee*®, and Young-Mock Lee ${ }^{\oplus}$ \\ Department of Pediatrics, Gangnam Severance Hospital, Yonsei University College of Medicine, Seoul, Korea
}

\begin{abstract}
The combination of central nervous system abnormalities and renal impairment is a notable characteristic of GallowayMowat syndrome (GAMOS), a disease which often accompanies microcephaly, developmental delay, and nephrotic syndrome. Many subtypes exist having various phenotypes and genotypes, and many genetic causes are still being identified. An 18-month-old boy first visited our clinic for seizure, delayed development, and microcephaly. During follow-up visits he developed proteinuria and nephrotic syndrome at the age of 6 . Nephrotic syndrome became refractory to treatment. These phenotypes were suggestive of GAMOS. Next generation sequencing was performed for genetic analysis and revealed novel compound heterozygous variants in the WDR4 gene: c.494G>A (p.Arg165Gln) and c.540C >G (p.lle180Met). This is the first case in Korea of GAMOS involving the WDR4 gene.
\end{abstract}

Key words: Galloway-Mowat syndrome, Microcephaly, Growth retardation, Cerebellar diseases, Nephrotic syndrome, WDR4.

\section{Introduction}

Galloway-Mowat syndrome (GAMOS) is a disease first reported in 1968, defined by a spectrum of neurodevelopmental anomalies and renal-glomerular disease [1]. Clinically, the syndrome is characterized by central nervous system (CNS) abnormalities such as microcephaly, developmental delay, seizures, and cerebellar atrophy. Renal features include proteinuria and nephrotic syndrome with variable age of onset, which are often refractory to treatment [2].

It is a phenotypically and genetically heterogenous disease, with subtypes 1 to 8 discovered so far [3]. Variants in the gene WDR73 were first identified in 2014, leading to the definition of subtype GAMOS1 [2]. In 2017, additional variants in LAGE3, OSGEP, TP53RK, and TRPKB were discovered as the genetic causes of GAMOS syndromes 2-5, respectively [4]. In 2018, Braun et al. [5] first reported the homozygous variants in the gene WDR4 as the cause of GAMOS6 (MIM 618347) in a case of an Indian family with neurologic and renal abnormalities. Previously, two cases in 2015 and 2018, respectively, reported WDR4 gene variants causing microcephaly and growth retardation, but neither exhibited renal features, which is one of the important clinical features of GAMOS $[5,6]$. Thus, up to date, only one case has been reported with WDR4 as the cause of GAMOS6 [5].

Here we report the first case in Korea of GAMOS6 with novel compound heterozygous WDR4 gene variants responsible for its cause.

\footnotetext{
Received: 15 September 2020, Revised: 28 October 2020, Accepted: 29 October 2020, Published: 31 December 2020

${ }^{*}$ Corresponding author: Hyunjoo Lee, M.D., Ph.D. (iD https://orcid.org/0000-0002-1432-0449

Department of Pediatrics, Gangnam Severance Hospital, Yonsei University College of Medicine, 211 Eonju-ro, Gangnam-gu, Seoul 06273, Korea.

Tel: +82-2-2019-3350, Fax: +82-2-2019-4881, E-mail: genejoo@yuhs.ac

Conflict of interest: The authors declare that they do not have any conflicts of interest.

(C) This is an open-access article distributed under the terms of the Creative Commons Attribution Non-Commercial License (http://creativecommons.org/licenses/by-nc/4.0/) which permits unrestricted non-commercial use, distribution, and reproduction in any medium, provided the original work is properly cited.

(c) Copyright 2020 by the Korean Society of Medical Genetics and Genomics 


\section{Case}

The patient was the second child of non-consanguineous parents. He was delivered by cesarean section weighing 1,420 g due to premature rupture of membrane at 30 weeks' gestation. He was hospitalized in the neonatal intensive care unit for 50 days due to apnea. Craniosynostosis was present at birth. He presented bilateral cryptorchidism and micropenis and had orchiopexy at 15 months. He showed severe developmental delays. He has achieved full head control at 10 months and rolled over at 11 months. He could sit down with support at 20months. At 18 months, first seizure occurred, but electroencephalographies showed no remarkable findings.

He repeated afebrile seizures and was referred to our clinic at the age of 27 months due to seizures and delayed development. Microcephaly and failure to thrive were present at the initial visit, with a head circumference of $39.5 \mathrm{~cm}$ at 27 months, which was below $3 \mathrm{rd}$ percentile. Height $(79 \mathrm{~cm})$ and weight $(7.5 \mathrm{~kg})$ were also below 3 rd percentile. Some dysmorphic features were present as well, such as narrow forehead, frontal bossing, and short neck. The child could not meet the age-appropriate developmental milestones. The patient could not sit without support and crawling was not possible. His hand grasp was weak, and the child could barely babble and could not say a single meaningful word. Initial laboratory tests showed no remarkable findings except for lactic acidosis. Thyroid function tests were within normal limits. Brain magnetic resonance imaging (MRI) showed cerebral atrophy, cerebellar atrophy along with thinning of cor-
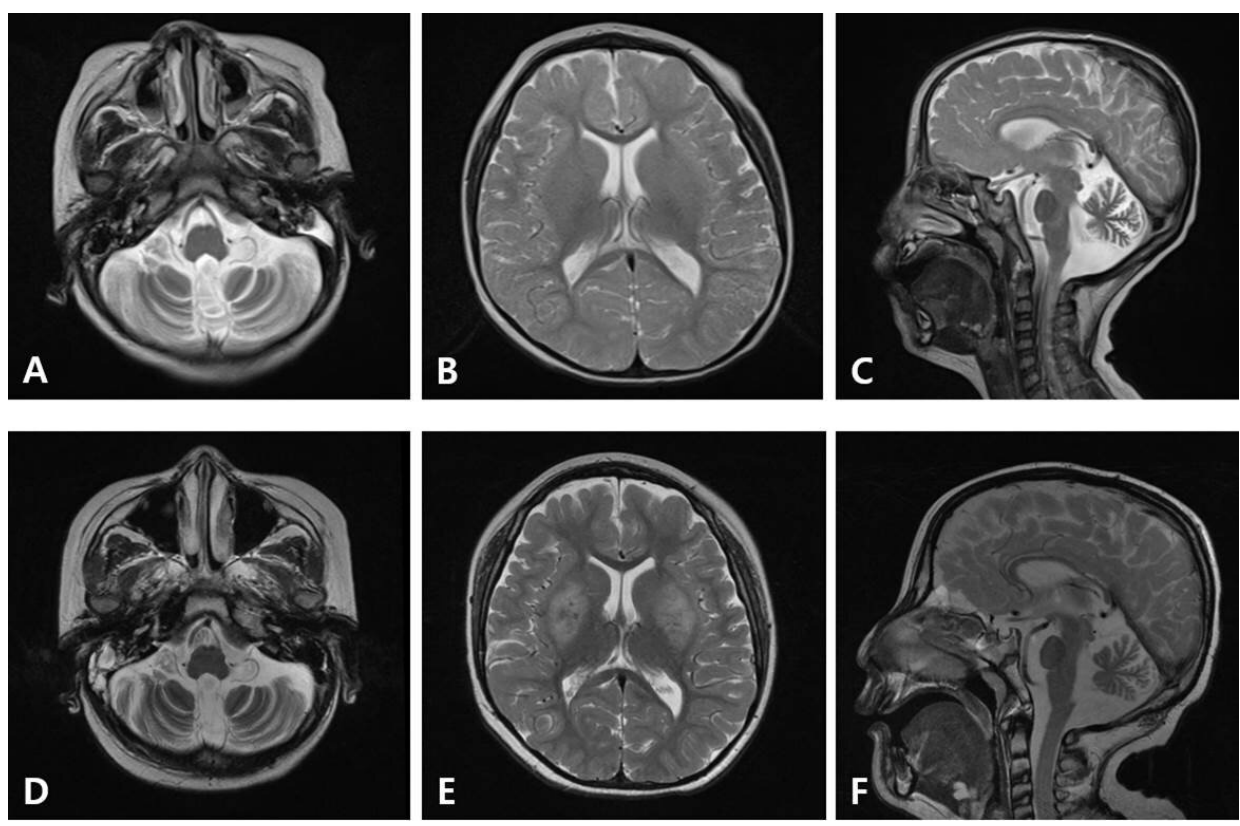

Fig. 1. T2-weighted magnetic resonance imaging of Galloway-Mowat syndrome with novel heterozygous WDR4 variants in a Korean boy at the age of 3 years $(A-C)$ and 9 years (D-F). (A) Cerebellar atrophy with mild edema was noted on axial view. (B) An axial image showed no significant signal intensity at both putamen. (C) A sagittal view showed cerebellar atrophy and cerebral atrophy with thinning of corpus callosum. (D) Cerebellar atrophy became more prominent compared to Fig. 1A. (E) An axial image showed newly developed hyperintensity at both putamen. (F) A sagittal view showed progressed cerebellar atrophy and thinning of corpus callosum.
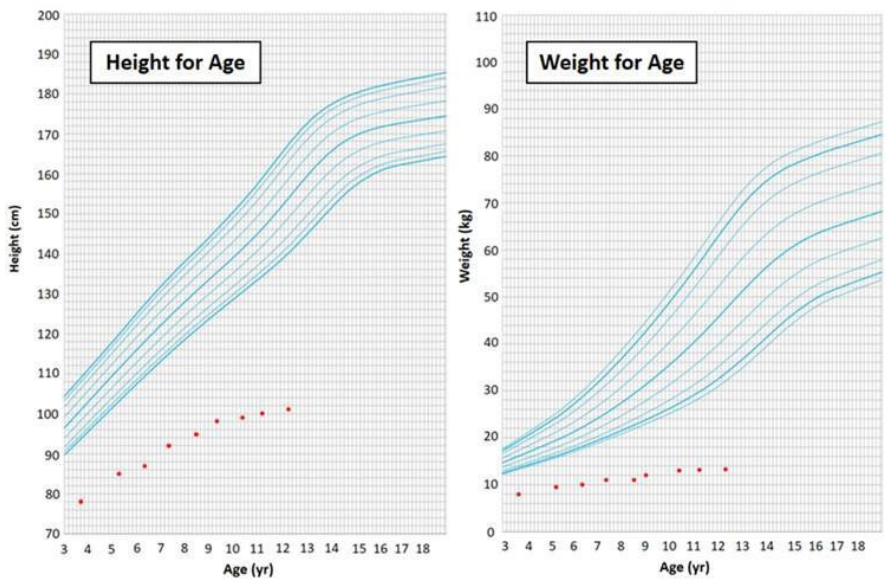

Age (yr)

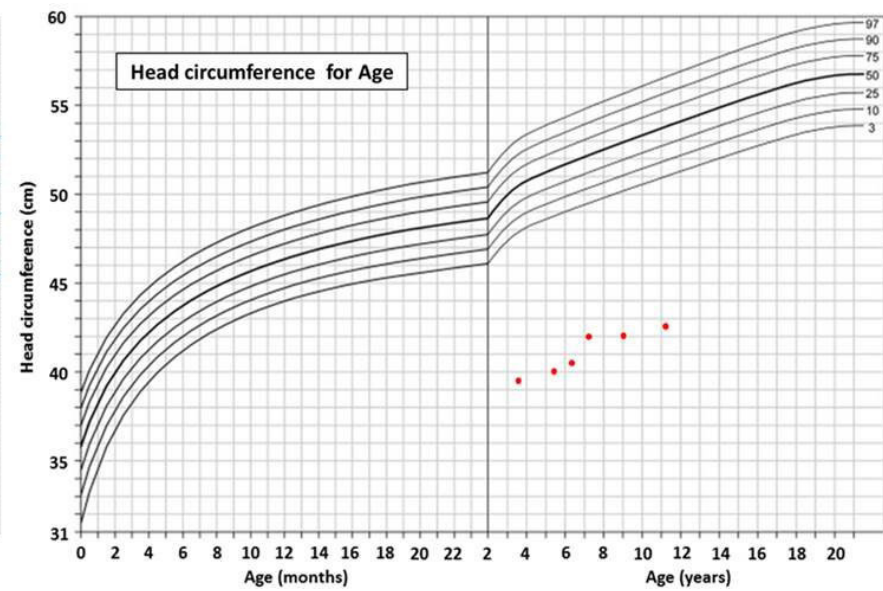

Fig. 2. Growth charts of the patient. 
pus callosum at 3 years of age (Figs. $1 \mathrm{~A}-\mathrm{C}$ ).

At 5 years, the patient developed occasional mild proteinuria $(1+)$ with no associated symptoms. And at the age of 6 years, he was admitted for lethargy and generalized edema accompanied by hypoalbuminemia $(2.5 \mathrm{mg} / \mathrm{dL})$, proteinuria $(3+)$ and increased urine protein-to-creatinine ratio (3.0). He was referred to a pediatric nephrologist and was diagnosed with nephrotic syndrome. Steroid therapy was initiated and shown to be effective at first, but later its dependency required addition of an ACE inhibitor. Regardless of the medication, the patient's nephrotic syndrome showed repeated rounds of exacerbation and improvement over the years.

At 9 years, He still had severe growth retardation with a severe microcephaly; head circumference was $42 \mathrm{~cm}$ ( $<3$ percentile), height and weight were $95 \mathrm{~cm}(<3$ percentile) and $12 \mathrm{~kg}(<3$ percentile) (Fig. 2). Development was stagnated, with independent sitting still not being possible and first word yet to be spoken. A follow-up MRI showed the progressed cerebral and cerebellar atrophy, and hyperintensity at both putamen was newly found (Figs. 1D-F). Seizures continued, for which the patient was treated with anti-epileptic drugs. And nephrotic syndrome showed occasional exacerbation. We performed whole-exome sequencing in trios (proband, mother, and father) to determine the cause of his multi-organ manifestation.

We identified compound heterozygous variants in the WDR4 gene NM_033661:c.540C>G (p.lle180Met) and NM_033661:c.494G>A (p.Arg165GIn). The variants were classified as variants of uncertain significance according to the guidelines of the American College of Medical Genetics and
Genomics [7]. However, in silico analysis by SIFT predicted these variants were deleterious, and PolyPhen suggested the variants were probably damaging [8]. ExAc allele frequency for each variant was 0.0000. The arginine residue at codon 165 and the isoleucine residue at codon 180 were highly conserved throughout evolution (Fig. 3A). A segregation study showed both parents as carriers of the variant, thus leading to the diagnosis of novel compound heterozygous variants (Fig. 3B). The variants were confirmed by Sanger sequencing in proband and both parents, proving that WDR4 variants were responsible for the patient's phenotypes and attributable to GAMOS6.

After genetic diagnosis, the patient maintains the supportive cares on a multi-disciplinary approach: neurology, nephrology, endocrinology, ophthalmology, and nutrition. At the age of 12, seizure frequency remains unchanged, and nephrotic syndrome is controlled with steroid and ACE inhibitor. Growth retardation persists, with a height of $101 \mathrm{~cm}(<3$ percentile) and a weight of $13 \mathrm{~kg}$ (<3 percentile) (Fig. 2). Development milestones show no improvement.

This study was approved by the Institutional Review Board of Yonsei University Health System (3-2020-0374). Written informed consent was waived.

\section{Discussion}

The hallmark of GAMOS is its involvement in both the CNS and the kidneys. Since its first discovery in 1968, more than 60 cases have been reported, exhibiting a wide spectrum of clinical phenotypes [1,9].

\begin{tabular}{|c|c|c|c|c|c|c|c|c|c|c|c|c|c|c|c|c|c|}
\hline & \multicolumn{17}{|c|}{ p.Arg165Gln } \\
\hline Homo sapiens & W & $S$ & v & $\mathrm{R}$ & 1 & K & $E$ & D & $R$ & $D$ & A & $\mathrm{T}$ & L & 1 & $\mathrm{~F}$ & $\mathrm{R}$ & $\mathrm{D}$ \\
\hline Macaca mulatta & w & $\mathrm{s}$ & v & $R$ & 1 & K & $E$ & $\mathrm{D}$ & $R$ & $D$ & A & $\mathrm{T}$ & L & I & $\mathrm{F}$ & $R$ & $\mathrm{D}$ \\
\hline Mus musculus & W & $\mathrm{S}$ & v & $\mathrm{R}$ & 1 & K & $E$ & D & $R$ & $D$ & A & T & L & v & $\mathrm{F}$ & Q & D \\
\hline Canis lupus familiaris & W & $S$ & v & $\mathrm{R}$ & 1 & K & $E$ & D & $\mathrm{R}$ & $D$ & A & $\mathrm{T}$ & L & v & Y & $\mathrm{R}$ & D \\
\hline Elephas maximus & W & $S$ & v & $R$ & 1 & K & $E$ & D & $R$ & $D$ & A & $\mathrm{T}$ & L & v & Y & $\mathrm{R}$ & G \\
\hline Danio rerio & $\mathrm{F}$ & $\mathrm{S}$ & v & $R$ & 1 & K & $E$ & $D$ & R & $D$ & A & $T$ & 1 & 1 & Y & $\mathrm{K}$ & D \\
\hline & \multicolumn{17}{|c|}{$\begin{array}{c}\text { p.lle180Met } \\
\downarrow \\
\end{array}$} \\
\hline Homo sapiens & $T$ & $\mathrm{H}$ & G & L & c & $\mathrm{F}$ & S & $E$ & 1 & $S$ & $\mathrm{H}$ & $P$ & A & A & A & W & $\mathrm{s}$ \\
\hline Macaca mulatta & $T$ & $\mathrm{H}$ & $G$ & L & C & $F$ & $S$ & E & 1 & $S$ & $\mathrm{H}$ & $P$ & A & A & $A$ & w & $s$ \\
\hline Mus musculus & $T$ & $\mathrm{H}$ & G & L & C & $\mathrm{F}$ & S & E & 1 & $S$ & H & P & A & A & A & W & $\mathrm{s}$ \\
\hline Canis lupus familiaris & $T$ & $\mathrm{H}$ & G & L & C & $\mathrm{F}$ & S & E & I & $S$ & H & $P$ & A & A & A & w & $\mathrm{s}$ \\
\hline Elephas maximus & $T$ & $\mathrm{H}$ & G & L & C & $F$ & S & $E$ & I & $S$ & $\mathrm{H}$ & P & A & A & $\mathrm{L}$ & W & $\mathrm{s}$ \\
\hline Danio rerio & $\mathrm{T}$ & $\mathrm{H}$ & G & L & $C$ & $\mathrm{~F}$ & A & $\mathrm{Q}$ & 1 & $\mathrm{~N}$ & $Y$ & $P$ & $S$ & $R$ & $\mathrm{R}$ & $\mathrm{F}$ & S \\
\hline
\end{tabular}

Fig. 3. (A) Complete evolutionary conservation of arginine 165 and isoleucine 180 of WDR4 gene. (B) Family pedigree. The novel compound heterozygous variants in WDR4 gene were confirmed by Sanger sequencing of the patient and his parents. 
Neurologic manifestations varied in severity. Some children were able to attend school, though their scholastic performances were impaired, while others did not show any developmental progress [5]. Seizures were present in some patients but not all. However, all patients manifested microcephaly and global developmental delay, as in the patient of this case. Imaging studies showed cerebral atrophy, gyral abnormalities, and cerebellar atrophy in some patients.

Renal involvement in GAMOS is characterized by proteinuria. Severity and onset of symptoms may also vary, from congenital nephrotic syndrome to mild, persistent proteinuria [5]. Often nephrotic syndrome in GAMOS is resistant to steroid therapy and progresses to end-stage renal disease requiring dialysis [2]. Nephrotic syndrome in our patient was not controlled well, becoming refractory to treatment over time. Renal biopsy was not done in this case; however other studies revealed histological findings of minimal change disease, mesangial cell proliferation, mesangial sclerosis, and focal segmental glomerulosclerosis, suggesting histological heterogeneity [10].
The gene WDR4 encodes tRNA-methytransferase noncatalytic subunit WDR4, an enzyme which is required for the 7-methylguanosine modification of tRNA [11]. Phenotypic features of WDR4 gene variants include growth deficiency, microcephaly, developmental delay, and intellectual disability [5]. Previously, this gene was often known to be associated with primordial dwarfism with various phenotypes [12]. Recently, two independent publications reported autosomal recessive variants in WDR4 in patients with microcephalic primordial dwarfism, and microcephaly with growth retardation $[6,12]$. In 2018, Braun et al. [5] first reported a pathogenic WDR4 gene variant case with proteinuria and nephrotic syndrome. By inclusion of renal involvement, GAMOS, with its CNS and renal features, may comprise the phenotypic spectrum of pathogenic WDR4 gene variants [5]. Studies revealed that variants in genes involved in tRNA modifications, such as the KEOPS complex members, are responsible for the pathogenesis of GAMOS, and the fact that WDR4 encodes an enzyme required for tRNA modification adds support to these findings [4].

Table 1. Clinical features of WDR4-related GAMOS (in comparison with Braun et al. [5])

\begin{tabular}{|c|c|c|c|c|c|}
\hline- & Patient \# 1 & Patient \# 2 & Patient \# 3 & Patient \# 4 & Patient \#5 \\
\hline Sex & Male & $\mathrm{N} / \mathrm{A}$ & $\mathrm{N} / \mathrm{A}$ & Male & $\mathrm{N} / \mathrm{A}$ \\
\hline Age (yr) & 12 & 15 & 13 & 11 & 4 \\
\hline Weight & $13(-6 Z)$ & $18.9(-6 Z)$ & $15.1(-7 Z)$ & $13(-8 Z)$ & $7(-4 Z)$ \\
\hline Height & $101(-7 Z)$ & $122(-5 Z)$ & $105(-6 Z)$ & $98(-6 Z)$ & $69(-5 Z)$ \\
\hline \multicolumn{6}{|l|}{ Neurological } \\
\hline Intellectual disability & Severe & Moderate & Mild & Severe & Severe \\
\hline Microcephaly & + & + & + & + & + \\
\hline Seizures & + & - & - & - & - \\
\hline Brain imaging &,+ cerebellar atrophy & - & - &,+ normal & - \\
\hline \multicolumn{6}{|l|}{ Renal } \\
\hline Proteinuria & $+(3+)$ & $+(3+)$ & $+(2+)$ & $+(3+)$ & - \\
\hline Nephrotic syndrome & + & - & - & + & - \\
\hline Onset & $6 \mathrm{yr}$ & $\mathrm{N} / \mathrm{A}$ & $\mathrm{N} / \mathrm{A}$ & N/A & N/A \\
\hline Treatment response & Refractory & $\mathrm{N} / \mathrm{A}$ & $\mathrm{N} / \mathrm{A}$ & Partial refractory & $\mathrm{N} / \mathrm{A}$ \\
\hline \multicolumn{6}{|l|}{ Endocrinologic } \\
\hline Growth retardation & + & + & + & + & + \\
\hline TFT abnormalities & - & + & + & + & - \\
\hline \multicolumn{6}{|l|}{ Etc. } \\
\hline Cryptorchidism & + & - & - & - & - \\
\hline Variants & $\begin{array}{l}\text { c. } 540 C>G \\
\text { c. } 494 G>A \\
\text { compound heterozy- } \\
\text { gous variants }\end{array}$ & $\begin{array}{l}\text { c. } 454-2 A>C, \\
\text { homozygous } \\
\text { variants }\end{array}$ & $\begin{array}{l}\text { c. } 454-2 A>C, \\
\text { homozygous } \\
\text { variants }\end{array}$ & $\begin{array}{l}\text { c. } 454-2 A>C, \\
\text { homozygous } \\
\text { variants }\end{array}$ & $\begin{array}{l}\text { c. } 454-2 A>C, \\
\text { homozygous } \\
\text { variants }\end{array}$ \\
\hline References & Present study & $\begin{array}{l}\text { Braun et al. [5] } \\
\text { patient-22 }\end{array}$ & $\begin{array}{l}\text { Braun et al. [5] } \\
\text { patient-23 }\end{array}$ & $\begin{array}{l}\text { Braun et al. [5] } \\
\text { patient-21 }\end{array}$ & $\begin{array}{l}\text { Braun et al. [5] } \\
\text { patient-24 }\end{array}$ \\
\hline
\end{tabular}

GAMOS, Galloway-Mowat syndrome; TFT, thyroid function test; N/A, not applicable.

Clinical characteristics of five GAMOS patients with WDR4 mutation. 
The clinical phenotypic characteristics of this case in comparison with patients from a report by Braun et al. [5] are shown in Table 1. These five patients are so far all of the reported cases GAMOS with WDR4. All patients showed growth retardation, intellectual disability, microcephaly, and proteinuria. However, the severity of the phenotypes differed, such as mild to severe intellectual disability. For renal involvement, some had proteinuria only while others had nephrotic syndrome which was refractory to treatment. There are suggestions that some phenotypes (e.g. nephrotic syndrome) are age-dependent and may develop over time, which is supported by the fact that our patient did not develop proteinuria and nephrotic syndrome until the age of six [5].

In conclusion, our patient had clinical phenotypes that were attributable to GAMOS, which included microcephaly, developmental delay, seizure, and refractory nephrotic syndrome. The identified novel compound heterozygous WDR4 gene variants in this patient could contribute to GAMOS-related genotype phenotype expansion.

\section{References}

1. Galloway WH, Mowat AP. Congenital microcephaly with hiatus hernia and nephrotic syndrome in two sibs. J Med Genet 1968;5:319-21.

2. Colin E, Huynh Cong E, Mollet G, Guichet A, Gribouval O, Arrondel $C_{1}$ et al. Loss-of-function mutations in WDR73 are responsible for microcephaly and steroid-resistant nephrotic syndrome: GallowayMowat syndrome. Am J Hum Genet 2014;95:637-48.

3. El Younsi M, Kraoua L, Meddeb R, Ferjani M, Trabelsi M, Ouertani I, et al. WDR73-related Galloway Mowat syndrome with collapsing glomerulopathy. Eur J Med Genet 2019;62:103550.
4. Braun DA, Rao J, Mollet G, Schapiro D, Daugeron MC, Tan W, et al. Mutations in KEOPS-complex genes cause nephrotic syndrome with primary microcephaly. Nat Genet 2017;49:1529-38.

5. Braun DA, Shril S, Sinha A, Schneider R, Tan W, Ashraf S, et al. Mutations in WDR4 as a new cause of Galloway-Mowat syndrome. Am J Med Genet A 2018;176:2460-5.

6. Trimouille A, Lasseaux E, Barat P, Deiller C, Drunat S, Rooryck C, et al. Further delineation of the phenotype caused by biallelic variants in the WDR4 gene. Clin Genet 2018;93:374-7.

7. Richards S, Aziz N, Bale S, Bick D, Das S, Gastier-Foster J, et al.; ACMG Laboratory Quality Assurance Committee. Standards and guidelines for the interpretation of sequence variants: a joint consensus recommendation of the American College of Medical Genetics and Genomics and the Association for Molecular Pathology. Genet Med 2015;17:405-24.

8. Adzhubei IA, Schmidt S, Peshkin L, Ramensky VE, Gerasimova A, Bork P, et al. A method and server for predicting damaging missense mutations. Nat Methods 2010;7:248-9.

9. Ekstrand JJ, Friedman AL, Stafstrom CE. Galloway-Mowat syndrome: neurologic features in two sibling pairs. Pediatr Neurol 2012;47:12932.

10. Cohen AH, Turner MC. Kidney in Galloway-Mowat syndrome: clinical spectrum with description of pathology. Kidney Int 1994;45:1407-15.

11. Alexandrov A, Martzen MR, Phizicky EM. Two proteins that form a complex are required for 7-methylguanosine modification of yeast tRNA. RNA 2002;8:1253-66.

12. Shaheen $R$, Abdel-Salam GM, Guy MP, Alomar R, Abdel-Hamid MS, Afifi $\mathrm{HH}$, et al. Mutation in WDR4 impairs tRNA m(7)G46 methylation and causes a distinct form of microcephalic primordial dwarfism. Genome Biol 2015;16:210. 